\title{
Novo coronavírus e os riscos do isolamento social para os idosos: revisão integrativa
}

\section{New coronavirus and the risks of social isolation for the elderly: integrative review}

\section{Nuevo coronavirus y los riesgos de aislamiento social para los ancianos: revisión integradora}

Ana Luiza Ferreira Aydogdu ${ }^{1}$

DOI:

RESUMO

Objetivo: Investigar na literatura os riscos do isolamento social para a saúde dos idosos durante a pandemia causada pelo novo coronavírus. Metodologia: Revisão integrativa de artigos publicados no ano de 2020 através de buscas no Google Acadêmico, na Biblioteca Virtual em Saúde e na National Library of Medicine PubMed, a partir da pergunta norteadora: qual a abordagem da literatura sobre os riscos do isolamento social para a saúde dos idosos durante a pandemia causada pelo novo coronavírus? Resultados: 0 novo coronavírus (SARS-CoV-2) trouxe a necessidade de isolamento social, principalmente para grupos de risco, como idosos. Entretanto, nos oito artigos analisados observou-se que essa medida acarreta outras enfermidades físicas e/ou psicossociais. Considerações Finais: Ao proteger contra 0 novo coronavírus, além da saúde física é preciso atentar também para a saúde mental e social do idoso.

DESCRITORES:

Coronavírus; Geriatria; Idoso; Isolamento social.

Informações do Artigo:

Recebido em: 21/05/2020

Aceito em: 16/07/2020

\section{ABSTRACT}

Objective: To investigate in the literature the risks of social isolation for the health of the elderly during the pandemic caused by the new coronavirus. Methodology: Integrative review of articles published in 2020, through searches on Google Scholar, Virtual Health Library and National Library of Medicine - PubMed, based on the guiding

${ }^{1}$ Enfermeira. Mestre em Administração Hospitalar. Doutoranda em Administração em Enfermagem na Faculdade de Enfermagem Florence Nightingale da Universidade de Istambul - Cerrahpasa. Istanbul Universitesi - Cerrahpasa, Florence Nightingale Hemsirelik Fakultesi. Endereço: Abide-i-Hurriyet Cad. 34381 Posta kodu: 34510 - Sisli - Istanbul Turquia. Telephone number: +(90) 535637 0703. E-mail: analuizaferreira.aydogdu@ogr.iu.edu.tr. E-mails: analuizaferreira.aydogdu@ogr.iu.edu.tr, luizafl@gmail.com 
question: what is the literature approach on the risks of social isolation for the health of the elderly during the pandemic caused by the new coronavirus? Results: The new coronavirus (SARS-CoV-2) brought the social isolation, especially for groups at risk, such as the elderly. However, at the analysed articles it was observed that the measure causes other physical and/or psychosocial illnesses. Final Considerations: When protecting against the new coronavirus, in addition to physical health, it is also necessary to pay attention to mental and social health of the elderly.

DESCRIPTORS:

Coronavirus; Geriatrics; Aged; Social isolation.

\section{RESUMEN}

Objetivo: Investigar en la literatura los riesgos del aislamiento social para la salud de los ancianos durante la pandemia causada por el nuevo coronavirus. Metodología: Revisión integradora de artículos publicados en 2020 a través de búsquedas en Google Académico, Biblioteca Virtual de Salud y National Library of Medicine - PubMed, basada en la pregunta guía: ¿cuál es el enfoque de la literatura sobre los riesgos del aislamiento social para la salud de los ancianos durante la pandemia causada por el nuevo coronavirus? Resultados: El nuevo coronavirus (SARS-CoV-2) trajo la necesidad de aislamiento social, especialmente para grupos en riesgo, como los ancianos. Sin embargo, en los artículos analizados se observó que la medida causa otras enfermedades físicas y/o psicosociales. Consideraciones Finales: Al proteger contra el nuevo coronavirus, además de la salud física, también es necesario prestar atención a la salud mental y social de los ancianos.

DESCRIPTORES:

Coronavirus; Geriatría; Anciano; Aislamiento social.

\section{INTRODUÇÃO}

A doença causada pelo novo coronavírus (SARS-CoV-2) é altamente contagiosa, apresentando uma letalidade maior para alguns grupos, como idosos e portadores de doenças crônicas ${ }^{(1)}$.

O SARS-CoV-2 causa a doença chamada Corona Virus Disease (COVID-19), que surgiu na China e atualmente está presente em 188 países. No Brasil, até 0 dia 10 de julho foram contabilizados 1.755.779 casos da doença e 69.184 óbitos, de acordo com esses dados o país é o segundo no mundo em número de casos e óbitos, ficando atrás apenas dos Estados Unidos da América(1-2).

Uma das principais medidas de prevenção da COVID-19 é o isolamento social, que é recomendado principalmente para idosos e doentes crônicos, pois, ao redor do mundo, o número de óbitos devido à doença vem apresentando-se maior entre esses indivíduos ${ }^{(1,3-5)}$.

O SARS-CoV-2 representa um risco maior para idosos e para doentes crônicos, a incidência de doenças como diabetes e hipertensão arterial também é maior entre as pessoas dessa faixa etária. Como o corpo humano vai sofrendo mudanças com o passar dos anos, ocorre uma diminuição da capacidade do sistema imunológico de responder às enfermidades, o que leva ao aumento da incidência dessas doenças em pessoas com mais de 60 anos ${ }^{(1)}$.

$\mathrm{Na}$ Europa mais de 95\% das mortes por COVID-19 ocorreram entre pessoas com 60 anos ou mais. Nos Estados Unidos da América $80 \%$ das fatalidades foram entre adultos com 65 anos ou mais. No Brasil a faixa etária mais acometida é a de 70 a 79 anos com $24,3 \%$ de óbitos. Segundo o último 
boletim epidemiológico divulgado pelo Ministério da Saúde dentre as comorbidades mais frequentes entre os óbitos causados por COVID-19 estão as cardiopatias e o diabetes, sendo que a maioria das pessoas que vieram a óbito e apresentavam comorbidades tinham 60 anos ou mais(3-5).

Os índices de esperança de vida estão aumentando cada vez mais, nunca as pessoas viveram tão longamente como vivem hoje e segundo a Organização Mundial de Saúde (OMS) esses números continuarão sofrendo acréscimo nos próximos anos. Em 2050, a população mundial com mais de 60 anos deve chegar a 2 bilhões. O número de idosos aumentará tão rapidamente no Brasil, que o país terá pouco tempo para se adaptar a essa nova realidade(6). Hoje, mais de 28 milhões de pessoas, ou seja, cerca de $13 \%$ da população brasileira tem 60 anos ou mais $(7)$.

É preciso proteger as pessoas suscetíveis, entre elas os idosos, evitando que saiam às ruas e sejam infectados pelo SARS-CoV-2, entretanto, o confinamento domiciliar também traz riscos para a saúde física e mental desse grupo de pessoas(8-10).

Faz-se necessário identificar os riscos do isolamento social para os idosos, já que a medida tomada visando proteger, não deve levar ao surgimento de outras doenças aumentando assim o risco de morte para esse grupo de pessoas.

O SARS-CoV-2 trouxe um destaque para os idosos e consequentemente para a enfermagem geriátrica(11). Uma das medidas mais importantes para o controle da doença é a interrupção da transmissão, para tanto, é necessário curar os doentes e proteger especialmente os grupos suscetíveis. No que se refere ao idoso, a enfermagem além de cuidar daqueles que já estão doentes, é também responsável pela educação dos mesmos, visando assim a prevenção da COVID-19.

\section{Objetivo}

O presente estudo visa investigar na literatura os riscos do isolamento social para a saúde dos idosos durante a pandemia causada pelo novo coronavírus.

\section{METODOLOGIA}

Trata-se de uma revisão integrativa da literatura que se fundamenta na síntese das pesquisas disponíveis referentes ao tema estudado(12). Para selecionar os textos incluídos na pesquisa, foram utilizadas seis etapas de pesquisa: elaboração da pergunta norteadora, busca ou amostragem na literatura, coleta de dados, análise crítica dos artigos incluídos, discussão dos resultados e apresentação da revisão integrativa(13).

Na primeira etapa da pesquisa de acordo com o tema e o objetivo do estudo determinou-se a pergunta norteadora: qual a abordagem da literatura sobre os riscos do isolamento social para a saúde dos idosos durante a pandemia causada pelo novo coronavírus? 
Na segunda etapa foi realizada a busca por artigos sobre o tema nas seguintes bases de dados: Google Acadêmico, Biblioteca Virtual em Saúde (BVS) e National Library of Medicine - PubMed utilizando descritores selecionados através dos Descritores em Ciências da Saúde (DeCS) ou Medical Subject Headings (Mesh): "aged" AND "coronavirus" AND "geriatrics" AND "social isolation".

A busca foi feita entre artigos publicados no ano de 2020, que abrangessem a temática riscos do isolamento social para os idosos durante a pandemia causada pelo novo coronavírus; cujos textos completos e gratuitos estivessem disponíveis na internet em língua portuguesa, inglesa ou espanhola. $A$ pesquisa foi realizada no mês de julho de 2020.

Durante a terceira etapa foram definidas as informações a serem extraídas dos artigos selecionados que foram registradas no quadro de informações: referência, título, resultado e tipo de estudo. Na etapa seguinte foi feita análise crítica dos artigos selecionados.

$\mathrm{Na}$ quinta etapa foi realizada a interpretação dos resultados e na sexta e última etapa realizouse a apresentação da revisão e síntese do conhecimento, apontando os principais resultados obtidos através da análise dos artigos.

\section{RESULTADOS}

Foram encontrados 94 artigos, após a exclusão de seis textos duplicados o resultado diminuiu para 88. Feita a leitura de títulos e resumos foram excluídos 67 artigos por não corresponderem ao tema abordado. Os 21 artigos restantes foram lidos na íntegra e oito deles foram selecionados já que respondiam à pergunta norteadora, enquanto 13 textos foram excluídos, pois, relatavam medidas para que idosos aderissem ao isolamento, COVID-19 e riscos para idosos, métodos para amenizar as consequências do isolamento social para idosos, riscos do isolamento para a população em geral, entre outros. A compilação sintética dos artigos com os títulos das produções e principais conclusões encontrase demonstrada no quadro 1.

Quadro 1. Artigos sobre os riscos do isolamento social na saúde do idoso durante a pandemia causada pelo SARS-CoV-2 


\begin{tabular}{|c|c|c|c|c|}
\hline Referências & Título & Objetivos & Resultados & Tipo de Estudo \\
\hline $\begin{array}{l}\text { Elman A. et } \\
\text { al. }(2020)\end{array}$ & $\begin{array}{l}\text { Effects of the } \\
\text { COVID-19 } \\
\text { outbreak on elder } \\
\text { mistreatment and } \\
\text { response in New } \\
\text { York City: Inicial } \\
\text { lessons }\end{array}$ & $\begin{array}{lr}\text { Explorar } & 0 \\
\text { impacto } & \text { da } \\
\text { COVID-19 em } & \text { em } \\
\text { idosos vitimas } \\
\text { de maus-tratos e } \\
\text { em pessoas em } \\
\text { risco r de } \\
\text { vitimização }\end{array}$ & $\begin{array}{l}\text { Os autores afirmam que o } \\
\text { impacto do distanciamento } \\
\text { social é especialmente } \\
\text { problemático para os idosos, } \\
\text { pois, muitos já enfrentavam } \\
\text { problemas sociais como } \\
\text { isolamento e solidão. Além } \\
\text { disso, o isolamento social } \\
\text { representa distanciamento da } \\
\text { família, dos amigos, do acesso } \\
\text { aos locais que dão suportes } \\
\text { sociais e de saúde. Os autores } \\
\text { oferecem soluções criativas } \\
\text { iniciais partilhando experiências } \\
\text { visando ajudar a otimizar a } \\
\text { preparação e a antecipação de } \\
\text { possíveis desafios }\end{array}$ & Comentário \\
\hline $\begin{array}{l}\text { Plagg B. et } \\
\text { al. }(2020)\end{array}$ & $\begin{array}{l}\text { Prolonged social } \\
\text { isolation of the } \\
\text { elderly during } \\
\text { COVID-19: } \\
\text { Between benefit } \\
\text { and damage }\end{array}$ & $\begin{array}{l}\text { Relatar sobre os } \\
\text { benefícios } \\
\text { prejuízos do } \\
\text { isolamento } \\
\text { social para } \\
\text { idosos }\end{array}$ & $\begin{array}{l}\text { Segundo os autores além dos } \\
\text { problemas psicológicos devido } \\
\text { ao isolamento, a redução de } \\
\text { atividades físicas representa a } \\
\text { longo prazo um prejuízo para a } \\
\text { saúde. Isso se aplica não só aos } \\
\text { idosos residentes em casas de } \\
\text { repouso, mas também àqueles } \\
\text { que moram sozinhos. Aponta } \\
\text { para a necessidade de uma } \\
\text { abordagem holística, incluindo } \\
\text { gerenciamento de riscos de } \\
\text { ameaças agudas e crônicas, } \\
\text { além da manutenção da } \\
\text { dignidade na vida }\end{array}$ & Correspondência \\
\hline $\begin{array}{l}\text { Aprahami I., } \\
\text { Cesari } \quad \text { M. } \\
(2020)\end{array}$ & $\begin{array}{l}\text { Geriatric } \\
\text { syndromes and } \\
\text { SARS-CoV-2: } \\
\text { More than just } \\
\text { being old }\end{array}$ & $\begin{array}{l}\text { Relatar sobre } \\
\text { síndromes } \\
\text { geriátricas e } \\
\text { SARS-CoV-2 }\end{array}$ & $\begin{array}{l}\text { O isolamento social pode levar a } \\
\text { importantes problemas físicos e } \\
\text { mentais em indivíduos idosos, } \\
\text { como depressão, ansiedade e } \\
\text { descompensação de doenças } \\
\text { crônicas. Conclui que geriatras } \\
\text { podem contribuir para a } \\
\text { restauração dos valores } \\
\text { humanos através de suas } \\
\text { experiências em cuidar de } \\
\text { idosos }\end{array}$ & Editorial \\
\hline $\begin{array}{l}\text { Sepúlveda- } \\
\text { Loyola W et } \\
\text { al. (2020) }\end{array}$ & $\begin{array}{l}\text { Impact of social } \\
\text { isolation due to } \\
\text { COVID-19 on } \\
\text { health in older } \\
\text { people: mental } \\
\text { and physical } \\
\text { effects and } \\
\text { recommendations }\end{array}$ & $\begin{array}{l}\text { Analisar o } \\
\text { impacto do } \\
\text { isolamento } \\
\text { social durante a } \\
\text { pandemia de } \\
\text { COVID-19 na } \\
\text { saúde mental e } \\
\text { física de idosos } \\
\text { e as } \\
\text { recomendações } \\
\text { para pacientes, }\end{array}$ & $\begin{array}{l}\text { Participantes: } 20.069 \text { indivíduos } \\
\text { de dez artigos descritivos } \\
\text { transversais, que vivem em } \\
\text { comunidades não } \\
\text { institucionalizadas. Achados } \\
\text { confirmam que a saúde mental e } \\
\text { física dos idosos é } \\
\text { negativamente afetada durante o } \\
\text { distanciamento social. Os } \\
\text { principais resultados apontam } \\
\text { para ansiedade, depressão, }\end{array}$ & Revisão narrativa \\
\hline
\end{tabular}




\begin{tabular}{|c|c|c|c|c|}
\hline & & $\begin{array}{l}\text { cuidadores e } \\
\text { profissionais de } \\
\text { saúde }\end{array}$ & $\begin{array}{l}\text { pobre qualidade de sono e falta } \\
\text { de atividade física durante } 0 \\
\text { período de isolamento. } \\
\text { Considera sobre a importância } \\
\text { de um programa de exercícios e } \\
\text { estratégias psicológicas para a } \\
\text { população idosa durante } 0 \\
\text { confinamento }\end{array}$ & \\
\hline $\begin{array}{l}\text { Lim WS. } \\
\text { et al. (2020) }\end{array}$ & $\begin{array}{l}\text { COVID-19 and } \\
\text { older people in } \\
\text { Asia: Asian } \\
\text { working groups for } \\
\text { sarcopenia calls to } \\
\text { actions }\end{array}$ & $\begin{array}{lr}\text { Avaliar } & \text { os } \\
\text { impactos } & \text { da } \\
\text { COVID-19 } & \text { em } \\
\text { pessoas } & \text { idosas } \\
\text { de } & \text { países } \\
\text { asiáticos } & \end{array}$ & $\begin{array}{l}\text { As considerações do Grupo de } \\
\text { Trabalho Asiático para } \\
\text { Sarcopenia enfocando respostas } \\
\text { e dificuldades em cada país } \\
\text { apontam para consequências } \\
\text { adversas do isolamento social } \\
\text { entre idosos: solidão, redução do } \\
\text { acesso aos serviços básicos, } \\
\text { recaída física e cognitiva } \\
\text { gerando dependência. Conclui } \\
\text { que pandemia apresenta } \\
\text { desafios únicos podendo ser } \\
\text { uma oportunidade para os } \\
\text { países asiáticos redesenharem o } \\
\text { atendimento a essa população } \\
\text { vulnerável }\end{array}$ & Revisão literária \\
\hline $\begin{array}{l}\text { Azzolino D. } \\
\text { et al. }(2020)\end{array}$ & $\begin{array}{l}\text { Nutritional } \\
\text { considerations in } \\
\text { frail older patients } \\
\text { with COVID-19 }\end{array}$ & $\begin{array}{l}\text { Considerar } \\
\text { sobre as } \\
\text { condições } \\
\text { nutricionais de } \\
\text { pacientes idosos } \\
\text { fracos com } \\
\text { COVID-19 }\end{array}$ & $\begin{array}{l}\text { Autores relatam que além do } \\
\text { sentimento de solidão, o } \\
\text { isolamento social pode fazer } \\
\text { com que idosos não tenham } \\
\text { acesso aos supermercados } \\
\text { como tinham antes, a união } \\
\text { desses fatores pode levar à má } \\
\text { nutrição. Alerta para a } \\
\text { necessidade de avaliação do } \\
\text { estado nutricional em todos os } \\
\text { pacientes com COVID-19 na } \\
\text { admissão e durante a internação }\end{array}$ & Editorial \\
\hline $\begin{array}{l}\text { Kyriazis } \quad M . \\
(2020)\end{array}$ & $\begin{array}{l}\text { COVID-19 } \\
\text { isolation and risk } \\
\text { of death in elderly } \\
\text { people in Cyprus }\end{array}$ & \begin{tabular}{lr}
\multicolumn{3}{l}{ Relatar sobre os } \\
perigos & do \\
isolamento & \\
social para a & saúde \\
idosos, dos \\
estão em & casa \\
devido & à \\
pandemia & de \\
Covid-19 &
\end{tabular} & $\begin{array}{l}\text { O autor alerta que há uma } \\
\text { relação entre o isolamento social } \\
\text { e o risco de morte entre os } \\
\text { idosos. Além da solidão, a } \\
\text { redução do contato do idoso com } \\
\text { enfermeiros e médicos leva ao } \\
\text { aumento da mortalidade entre } \\
\text { eles. Aponta para a necessidade } \\
\text { de implementação de suporte } \\
\text { psicológico e médico, incentivo a } \\
\text { exercícios cognitivos e físicos } \\
\text { para idosos em suas residências }\end{array}$ & Relatório curto \\
\hline $\begin{array}{l}\text { Freitas AVS. } \\
(2020)\end{array}$ & $\begin{array}{l}\text { Instituições de } \\
\text { longa } \\
\text { permanência para } \\
\text { idosos e a COVID- } \\
\text { 19: urgência no } \\
\text { debate }\end{array}$ & $\begin{array}{l}\text { Apresentar uma } \\
\text { breve reflexão } \\
\text { sobre a urgência } \\
\text { no debate em } \\
\text { relação às } \\
\text { instituições de } \\
\text { longa }\end{array}$ & $\begin{array}{l}\text { Achados obtidos através de } \\
\text { revisão não sistemática } \\
\text { confirmam que a suspensão do } \\
\text { contato dos idosos com a família } \\
\text { nas instituições de longa } \\
\text { permanência favorece a } \\
\text { intensificação da sensação de }\end{array}$ & $\begin{array}{l}\text { Estudo descritivo, } \\
\text { reflexivo }\end{array}$ \\
\hline
\end{tabular}




\begin{tabular}{|c|c|c|c|}
\hline & & $\begin{array}{l}\text { permanência } \\
\text { para Idosos e a } \\
\text { COVID-19 }\end{array}$ & $\begin{array}{l}\text { isolamento, insegurança e } \\
\text { solidão, gerando preocupação } \\
\text { com a saúde dos familiares; } \\
\text { além das preocupações com a } \\
\text { própria vida e o medo da morte e } \\
\text { do abandono. Pondera sobre a } \\
\text { diversidade existente nas } \\
\text { instituições de longa } \\
\text { permanência, no que diz respeito } \\
\text { aos residentes, estrutura física e } \\
\text { capacidade dos profissionais em } \\
\text { cuidar, com base nas } \\
\text { especificidades do } \\
\text { envelhecimento para evitar que } \\
\text { pacientes seiam afetados }\end{array}$ \\
\hline
\end{tabular}

Fonte: Elaborado pela autora, 2020

Sete artigos em inglês fazem parte de seis publicações internacionais diferentes, um artigo em português foi publicado em periódico nacional: Journal of Applied Gerontology, Archives of Gerontology and Geriatrics, The Journal of Frailty \& Aging Clinical, The Journal of Nutrition Health and Aging, Geriatrics \& Gerontology International, National Gerontology Centre e Research, Society and Development. No que diz respeito à metodologia são dois editoriais, um comentário, uma correspondência, um relatório curto, uma revisão literária, uma revisão narrativa e um estudo descritivo, reflexivo.

\section{DISCUSSÃO}

Entre as principais medidas de prevenção da COVID-19 estão o distanciamento e o isolamento social. Recomenda-se principalmente que pessoas de grupos de risco evitem sair de seus domicílios, visando assim diminuir os riscos de contágio(14).

Após a análise detalhada dos artigos, constatou-se que, o isolamento social pode trazer consequências negativas tanto para a saúde física quanto para a saúde mental e social dos idosos ${ }^{(9-10,15-}$ 20).

Ao serem isolados os idosos são protegidos contra o SARS-CoV-2, mas muitos deles são contrários ao isolamento, além disso, essa medida pode levar ao surgimento de distúrbios físicos e psicológicos(9-10). Governos de vários países pedem que idosos permaneçam em seus domicílios, fornecem ajuda quanto à compra de supermercado e farmácia(14), entretanto, faz-se necessário lembrar que saúde é um estado de bem-estar físico, psicológico e social.

É importante que a capacidade funcional dos idosos seja mantida, por isso atividades físicas devem ser praticadas diariamente. 0 sedentarismo do idoso favorecerá ao surgimento de doenças e 0 agravamento de enfermidades preexistentes $(6-9,17)$. 
O isolamento representa um risco também para a saúde mental do idoso, podendo levar ao surgimento de ansiedade, medo e depressão. $\mathrm{O}$ idoso em isolamento pode sentir-se abandonado e desmotivado, o que poderá gerar conflitos familiares. Além disso, todos esses fatores afetarão negativamente seu sistema imunológico, deixando-o ainda mais propenso ao adoecimento(8-10,14-15,18).

Os idosos fazem parte de um grupo de pessoas que normalmente já se sentiam isolados mesmo antes do surgimento do SARS-CoV-2. O distanciamento de familiares e amigos, e a redução do acesso aos serviços básicos causou um aumento dos níveis de ansiedade, insegurança, medo e depressão nas pessoas idosas, o que pode levar a recaídas físicas e cognitivas fazendo com que fiquem cada vez mais dependentes $(9,15,17-18)$.

O ser humano é um ser social, que precisa estar em contato com outros para sentir-se saudável, o idoso necessita não só de apoio no que se refere à sua condição física, mas também deve ser considerada sua saúde psicossocial.

É necessário que a pessoa idosa seja vista com um ser integral, que deve ser estimulado para ter qualidade de vida, a mais importante forma de estímulo é a comunicação(21).

As pessoas em isolamento estão recorrendo à tecnologia visando amenizar o sentimento de solidão e também diminuir o estresse que a situação atual vem provocando. Muitos dos idosos não tem acesso à internet e smartphones, muitos deles não sabem nem como utilizá-los, para eles a socialização ocorre nas pracinhas, centros comunitários e igrejas. Medidas devem ser planejadas visando diminuir as tensões desse grupo em especial(8,14-15).

A solidão e a depressão podem fazer com que o idoso não sinta vontade de se alimentar, esse fato adicionado à diminuição ao acesso aos supermercados pode acarretar má nutrição do idoso deixando-o ainda mais exposto a doenças ${ }^{(10)}$. Além disso, ansiedade, sentimento de abandono, diminuição da qualidade do sono, que aumentaram devido ao isolamento social, associados ao distanciamento dos idosos de seus médicos e enfermeiros acaba aumentando o risco de morte entre esse grupo de pessoas ${ }^{(17,19)}$.

Outro fator que deve ser considerado é a situação daqueles que residem em casas de repouso. Nessas instituições o risco de infecção por COVID-19 é grande, pois, muitas pessoas que fazem parte de grupos de risco vivem num mesmo ambiente. Tais estabelecimentos não têm estrutura adequada para cuidar de casos graves de COVID-19, não dispondo de equipamentos, nem de pessoal com formação para tal. Pacientes serão levados para o hospital mais próximo, o que em um breve período levará à estagnação dessa instituição de saúde. Outra desvantagem da casa de repouso em tempos de pandemia de COVID-19 é o fato dos idosos não poderem receber visitas de familiares e amigos, o que faz com que se sintam cada vez mais solitários e deprimidos. $O$ isolamento social gerou ainda a preocupação com familiares além de ter aumentado o medo do abandono e da morte entre os idosos residentes em casas 
de repouso $(11,16,20,22)$.

A população precisa ser bem orientada sobre a importância de proteger grupos de risco. 0 isolamento social do idoso precisa ser feito de forma zelosa para que ele não se sinta privado de sua liberdade e sim cuidado pela família e pela comunidade.

A comunicação correta também é de suma importância para que a comunidade e o próprio idoso entendam porque ele se encontra em um grupo de risco e, porque deve optar por medidas de isolamento social. Informações equivocadas resultaram em julgamentos errôneos que provocaram situações de preconceito, discriminação e falta de respeito com idosos.

Ao redor do mundo, visando reduzir o contágio da COVID-19 foi aconselhado 0 isolamento social, principalmente para pessoas de grupos de risco. No Brasil, além dessa medida, antecipou-se a vacinação contra influenza, esse fato fez com que idosos em isolamento saíssem de suas residências e lotassem postos de saúde. Aqui, mais uma vez fica nítida a necessidade de divulgação de informações de forma correta(8,11).

Num momento em que o idoso se tornou o centro das atenções ficou evidente também a importância da enfermagem geriátrica. Com a necessidade de medidas apropriadas visando proteger e educar o idoso, a figura do enfermeiro mais uma vez se faz presente, pois, além de ser um cuidador esse profissional é também um educador(11).

Ao cuidar do idoso em tempos de COVID-19, a equipe de enfermagem deve ser ainda mais cautelosa, assistindo e atentando para possível piora daqueles que já estão infectados, dando especial atenção às características peculiares desse grupo de pessoas; reconhecendo sintomas naqueles que ainda não foram diagnosticados e tomando todas as medidas para controle da infecção.

Informar ao idoso e à comunidade de forma clara, concisa e respeitosa sobre a doença e formas de prevenção fará com que a pessoa idosa não se sinta abandonada devido às medidas de isolamento e sim protegida e valorizada. 0 idoso deve ser orientado com relação à proteção também de sua saúde mental e social durante o isolamento, para que essa medida não venha agravar seu estado de saúde.

\section{Limitações do Estudo}

Considera-se como limitação o fato de nos resultados das buscas por artigos não ter sido identificado nenhum artigo original por se tratar de uma temática recente.

\section{Contribuições para a Área da Enfermagem, Saúde ou Política Pública}

Para deter a pandemia causada pelo SARS-CoV-2 uma das medidas mais importantes é a proteção dos grupos suscetíveis. Idosos fazem parte do grupo de risco, por isso é importante conhecer 
as características peculiares dessa fase da vida, educando não somente eles, mas toda a população com relação à prevenção da doença, o que implica a proteção correta das pessoas idosas. As medidas protetivas devem ser tomadas de forma cautelosa para que não acarretem outros problemas físicos ou mentais aos idosos. 0 enfermeiro além de cuidador é também pesquisador e educador, tendo papel fundamental no controle da COVID-19, não só tratando dos doentes, mas também oferecendo informações corretas sobre a doença e as devidas medidas preventivas.

\section{CONSIDERAÇÕES FINAIS}

Ao proteger o idoso contra o SARS-CoV-2 mantendo-o no domicílio, é preciso considerar que esse fato poderá acarretar problemas para sua saúde física, mental e social. O idoso não deve se sentir abandonado por seus familiares e amigos. É preciso explicar os motivos do isolamento para que ele se sinta valorizado e não discriminado. Deve-se atentar para mudanças nas condições físicas e mentais do idoso, como ansiedade, insegurança, medo, depressão, insônia, falta de apetite, cansaço entre outras. Faz-se necessário desenvolver sistemas de apoio físico, mental e social à distância que possam ser efetivamente utilizados pelos idosos.

Atividades físicas devem ser realizadas pelas pessoas da terceira idade e deve-se buscar formas alternativas de entretenimento para proteger também a saúde mental e social desse grupo de pessoas. O isolamento não deve impedir que o idoso tenha acesso aos remédios de rotina, devendo ser auxiliado quanto ao assunto. Deve ser dada atenção à alimentação, hidratação e higiene correta da pessoa idosa, visando fortalecer seu aparelho imunológico.

O sistema de saúde, bem como a equipe de enfermagem devem estar preparados para cuidar do idoso em todos os níveis de assistência, tratando o idoso doente, educando-o quanto às formas de prevenção contra à COVID-19 e ao reconhecimento de agravos físicos e mentais que podem surgir devido ao isolamento social. As peculiaridades desse grupo de pessoas devem ser reconhecidas proporcionando-lhes qualidade de vida. Proteger o idoso significa proteger também o sistema de saúde contra uma estagnação sem precedentes.

\section{REFERÊNCIAS}

1. Flores TG, Lampert MA. Por que idosos são mais propensos a eventos adversos com a infecção por COVID-19?. Revista Amazonense de Geriatria e Gerontologia. [Internet]. 2020 [acesso em 2020 mai 21]; especial Covid-19 e coronavírus. Disponível em: https://raggfunati.com.br/docs/covid/Flores\%20e\%20Lampert.pdf.

2. Johns Hopkins University. COVID-19 Dashboard by the Center for Systems Science and Engineering (CSSE). [Internet]. 2020 [cited 2020 Jun 05]. Available from: https://coronavirus.jhu.edu/map.html 
3. Centers for Disease Control and Prevention. Coronavirus disease 2019 (COVID-19). [Internet]. 2020 [cited 2020 Jul 10]. Available from: https://www.cdc.gov/coronavirus/2019-ncov/need-extraprecautions/older-adults.html.

4. World Health Organization (WHO). Statement - Older people are at highest risk from COVID-19, but all must act to prevent community spread. 2020 [cited 2020 Jul 10]. Available from: https://www.euro.who.int/en/health-topics/health-emergencies/coronavirus-covid19/statements/statement-older-people-are-at-highest-risk-from-covid-19,-but-all-must-act-toprevent-community-spread.

5. Brasil. Ministério da Saúde, Secretaria de Vigilância em Saúde. 20 Boletim Epidemiológico Especial, Doença pelo Coronavírus COVID-19. [Internet]. Brasília, DF: MS; 2020 [acesso em 2020 jul 10]. Disponivel em: http://saude.gov.br/images/pdf/2020/July/01/Boletim-epidemiologico-COVID-203.pdf.

6. World Health Organization (WHO). Ageing and health. [Internet]. 2018 [cited 2020 May 19]. Available from: https://www.who.int/news-room/fact-sheets/detail/ageing-and-health.

7. Benecdito, M. (Ed.). Longevidade, viver bem e cada vez mais. Retratos a Revista do IBGE. [Internet]. Rio de Janeiro, Brasil; 2019 [acesso em 2020 mai 19]. Disponível em: https://agenciadenoticias.ibge.gov.br/media/com_mediaibge/arquivos/d4581e6bc87ad8768073f974 c0a1102b.pdf.

8. World Health Organization (WHO). Mental health and psychosocial considerations during the COVID-19 outbreak. [Internet]. 2020 [cited 2020 May 21]. Available from: https://www.who.int/docs/default-source/coronaviruse/mental-health-considerations.pdf.

9. Aprahamian I, Cesari M. Geriatric syndromes and SARS-COV-2: More than just being old. Journal of Frailty \& Aging. [Internet]. 2020 [cited 2020 Jul 08]; 9(3):127-129 Available from: https://www.ncbi.nlm.nih.gov/pmc/articles/PMC7196375/.

10. Azzolino D, Saporiti E, Proietti M, Cesari M. Nutritional considerations in frail older patients with COVID-19. Journal or Nutrition Health and Aging. [Internet]. 2020 [cited 2020 Jul 08]. Available from: https://www.ncbi.nlm.nih.gov/pmc/articles/PMC7256177/.

11. Hammerschmidt KSA, Santana RF. Saúde do idoso em tempos de pandemia Covid-19. Cogitare enferm. [Internet]. 2020 [acesso em 2020 mai 19]. Disponível em: http://docs.bvsalud.org/biblioref/2020/04/1095404/72849-288133-1-pb.pdf.

12. Souza MT, Silva MD, Carvalho R. Revisão integrativa: o que é e como fazer. Einstein. [Internet]. 2010 [acesso em 2020 jul 07]; 8(1 Pt 1): 102-6. Disponível em: https://www.scielo.br/pdf/eins/v8n1/pt_1679-4508-eins-8-1-0102.pdf.

13. Ganong LH. Integrative reviews of nursing research. Res Nurs Health. 1987;10(1):1-11. 
14. Armitage R, Nellums LB. COVID-19 and the consequences of isolating the elderly. Lancet. [Internet]. 2020 [cited 2020 May 19]; 5(1):e506. Available from: https://doi.org/10.1016/ S2468-2667(20)30061$X$.

15. Elman A, Breckman R, Clark S, Gottesman E, Rachmuth L, Reiff M, et al. Effects of the COVID 19 outbreak on elder mistreatment and response in New York City: Initial lessons. Journal of Applied Gerontology. [Internet]. 2020 [cited 2020 Jul 08]; 39(7): 690 699. Available from: https://journals.sagepub.com/doi/full/10.1177/0733464820924853

16. Plagg B, Engl A, Piccoliori G, Eisendle K. Prolonged social isolation of the elderly during COVID-19: Between benefit and damage. Archives of Gerontology and Geriatrics. [Internet]. 2020 [cited 2020 Jul 08]; 89(2020):104086. Available from: https://www.ncbi.nlm.nih.gov/pmc/articles/PMC7196375/.

17. Sepúlveda-Loyola W, Rodriguéz-Sánchez I, Pérez-Rodríguez P, Ganz F, Torralba R, Oliveira DV at al. Impact of social isolation due to COVID-19 on health in older people: Mental and physical effects and recommendations. Journal of Nutrition Health and Aging. [Internet]. 2020 [cited 2020 Jul 08]. Available from: https://www.researchgate.net/publication/342278319.

18. Lim WS, Liang CK, Assantachai P, Auyeung TW, Kang L, Lee WJ et al. COVID-19 and older people in Asia: Asian working group for sarcopenia calls to actions. Geriatrics \& Gerontolgy International. [Internet]. 2020 [cited 2020 Jul 08]; 20(6):547-558 Available from: https://pubmed.ncbi.nlm.nih.gov/32365259/.

19. Kyriazis M. COVID-19 isolation and risk of death in elderly people in Cyprus. Short Report. National Gerontology Centre. [Internet]. 2020 [cited 2020 Jul 08]. Available from: https://www.researchgate.net/publication/340600133_COVID19_Isolation_and_Risk_of_Death_in_Elderly_People_in_Cyprus_Short_Report_National_Gerontology_Centre_Cyprus_April_2020.

20. Freitas AVS. Instituições de longa permanência para idosos e a covid-19: urgência no debate. Research, Society and Development. [Internet]. 2020 [acesso em 2020 jul 07]; 9(7):1-11, e490974398. Disponível em: http://dx.doi.org/10.33448/rsd-v9i7.4398.

21. Paschoallin HC, Perensim K. A importância da comunicação e do estímulo para a qualidade de vida do idoso. Rev. Enf. UFJF. [Internet]. 2015 [acesso em 2020 mai 20]; 1(1):45-53. Disponível em: https://periodicos.uffi.br/index.php/enfermagem/article/view/3787.

22. Gardner W, States D, Bagley N. The coronavirus and the risks to the elderly in long-term care. Journal of Aging \& Social Policy. [Internet]. 2020 [cited 2020 May 20]. Available from: https://www.tandfonline.com/doi/full/10.1080/08959420.2020.1750543? scroll=top\&needAccess=tru e. 